Journal of Magnetic Resonance 234 (2013) 1-9

http://dx.doi.org/10.1016/j.jmr.2013.06.002 (†)

\title{
Multi-frequency EDMR applied to microcrystalline thin-film silicon solar cells
}

\author{
Christoph Meier ${ }^{\mathrm{a}}$, Jan Behrends ${ }^{\mathrm{a}, *}$, Christian Teutloff ${ }^{\mathrm{a}}$, Oleksandr \\ Astakhov $^{\mathrm{b}}$, Alexander Schnegg ${ }^{\mathrm{c}}$, Klaus Lips ${ }^{\mathrm{c}}$, Robert Bittl ${ }^{\mathrm{a}}$ \\ ${ }^{a}$ Freie Universität Berlin, Fachbereich Physik, Arnimallee 14, D-14195 Berlin, Germany \\ ${ }^{b}$ Forschungszentrum Jülich, Institut für Energieforschung - Photovoltaik, D-52425 \\ Jülich, Germany \\ ${ }^{c}$ Helmholtz-Zentrum Berlin für Materialien und Energie, Institut für \\ Silizium-Photovoltaik, Kekuléstr. 5, D-12489 Berlin
}

\begin{abstract}
Pulsed multi-frequency electrically detected magnetic resonance (EDMR) at X-, Q- and W-Band (9.7, 34, and $94 \mathrm{GHz}$ ) was applied to investigate paramagnetic centers in microcrystalline silicon thin-film solar cells under illumination. The EDMR spectra are decomposed into resonances of conduction band tail states (e states) and phosphorus donor states (P states) from the amorphous layer and localized states near the conduction band (CE states) in the microcrystalline layer. The e resonance has a symmetric profile at all three frequencies, whereas the CE resonance reveals an asymmetry especially at $\mathrm{W}$-band. This is suggested to be due to a size distribution of $\mathrm{Si}$ crystallites in the microcrystalline material. A gain in spectral resolution for
\end{abstract}

\footnotetext{
*mail: j.behrends@fu-berlin.de, phone: 0049 (0)30 83852769

Email addresses: christoph.meier@fu-berlin.de (Christoph Meier), j.behrends@fu-berlin.de (Jan Behrends), christian.teutloff@fu-berlin.de (Christian Teutloff), o.astakhov@fz-juelich.de (Oleksandr Astakhov), alexander.schnegg@helmholtz-berlin.de (Alexander Schnegg), lips@helmholtz-berlin.de (Klaus Lips), robert.bittl@fu-berlin.de (Robert Bittl)
}

$(\dagger)$ Disclaimer: The publisher's version overrides in case of any discrepancy. 
the e and CE resonances at high fields and frequencies demonstrates the advantages of high-field EDMR for investigating devices of disordered Si. The microwave frequency independence of the EDMR spectra indicates that the same spin polarization-independent transport process is responsible for the EDMR signals observed at X-, Q- and W-band.

Keywords:

electron spin resonance, electrically detected magnetic resonance, multi-frequency EDMR, microcrystalline silicon, solar cells

\section{Introduction}

Thin-film solar cells bear the potential of converting solar energy at much lower costs than the well established wafer-based technology. Among the various materials used in thin-film solar cells, hydrogenated amorphous silicon (a-Si:H) and hydrogenated microcrystalline silicon ( $\mu \mathrm{c}-\mathrm{Si}: \mathrm{H})$ play a key role. Both materials can directly be deposited from the gas phase using lowtemperature processes on inexpensive substrates and require only a fraction of the material that is necessary for crystalline silicon (c-Si) solar cells [1-4]. By choice of the deposition parameters a-Si:H and $\mu \mathrm{c}-\mathrm{Si}: \mathrm{H}$ can be produced with the same deposition equipment and can thus easily be combined in one device. This allows for the fabrication of tandem solar cells utilizing the different band gaps of a-Si:H and $\mu \mathrm{c}-\mathrm{Si}: \mathrm{H}$ and, consequently, a more efficient use of the solar spectrum.

Since the fabrication of the first a-Si:H solar cell in 1976 [5], the maximum conversion efficiencies increased steadily to more than $12 \%$ for a-Si:H/ $\mu$ c$\mathrm{Si}: \mathrm{H}$ tandems $[6,7]$. However, this value is well below the maximum effi- 
ciencies achieved with c-Si-based cells [8]. This gap is due to the inferior electrical properties of amorphous thin-film silicon as compared to its crystalline counterpart. Deep defects due to dangling bonds (db) and localized tail states in the band gap induced by strained bonds act as recombination centers and traps, respectively, and thereby influence the charge transport of photogenerated charge carriers. They thus diminish the performance of the cell. Understanding these loss mechanisms on a microscopic level is a necessary prerequisite for the development of advanced cell concepts minimizing the detrimental influence of defect states on the cell performance.

Whenever a localized defect state is occupied by an odd number of electrons, it is paramagnetic and can be detected by electron paramagnetic resonance (EPR) spectroscopy. EPR can determine defect concentrations and thus allows for an optimization of a-Si:H and $\mu \mathrm{c}-\mathrm{Si}: \mathrm{H}$ deposition parameters [9]. Since the electron spin trapped in a defect state constitutes a sensitive probe for electron and nuclear spins in its vicinity, EPR and related multiresonance techniques can deliver helpful information on defect structures in solar cell materials [10-12]. In particular a multi-frequency approach has recently proven successful for the investigation of the EPR parameters of dbs in a-Si:H [13].

Whether these paramagnetic defects are indeed involved in trapping and recombination processes can conveniently be analyzed when combining EPR with a photocurrent measurement. The resulting technique, electrically detected magnetic resonance (EDMR) [14], interferes with spin-dependent recombination or hopping rates and probes the resulting change in conductivity. EDMR employing continuous wave (cw) microwave (mw) excitation has been 
applied to thin-film silicon films and devices for a couple of years [15]. More recently the combination of electrical detection with pulsed mw excitation schemes was shown to provide new insight into charge carrier recombination and hopping transport in thin-film silicon devices $[16,17]$. On the one hand, exploiting the time regime enabled the discrimination between spectrally overlapping EDMR signals associated with different spin-dependent processes in a-Si:H- and $\mu \mathrm{c}-\mathrm{Si}: \mathrm{H}-$ based solar cells $[18,19]$. On the other hand, pulsed EDMR has paved the way for the application of sophisticated detection schemes known from mw-detected EPR [20-23].

The present study is an extension of previous pEDMR work performed on $\mu \mathrm{c}-\mathrm{Si}: \mathrm{H}$ p-i-n thin-film solar cells $[18,19]$. Based on X-band pEDMR at cryogenic temperatures three resonances in the $\mu \mathrm{c}-\mathrm{Si}: \mathrm{H}$ pEDMR spectrum could be identified (Fig. 1). First, a signal at $g_{\mathrm{CE}}=1.9975(5)$ (CE signal) from shallow localized states in energetic proximity to the $\mu \mathrm{c}$-Si:H conduction band. This signal could be further attributed to states within $\mu \mathrm{c}-\mathrm{Si}: \mathrm{H}$ grains by electrically detected ESEEM [23]. Second, a resonance at $g_{\mathrm{e}}=2.0049(5)(\mathrm{e}$ signal) originating from n-a-Si:H conduction band tail states. Third, doublet signals of $\mathrm{P}$ donor states in n-a-Si:H. These signals are centered around $g_{\mathrm{P}} \approx$ 2.003(1) (P signal)) and exhibit a ${ }^{31} \mathrm{P}$ hyperfine splitting $(I=1 / 2$, natural abundance $100 \%$ ) of $\approx 25 \mathrm{mT}$.

All three signals were assigned to spin-dependent hopping processes in the $\mu \mathrm{c}-\mathrm{Si}: \mathrm{H}$ and n-a-Si:H layers. Since the energetic position of the quasi Fermi levels under the experimental conditions used here is supposed to be in the range of the energy band tail states, electrical transport is strongly influenced by hopping of charge carriers along localized states. No indications 


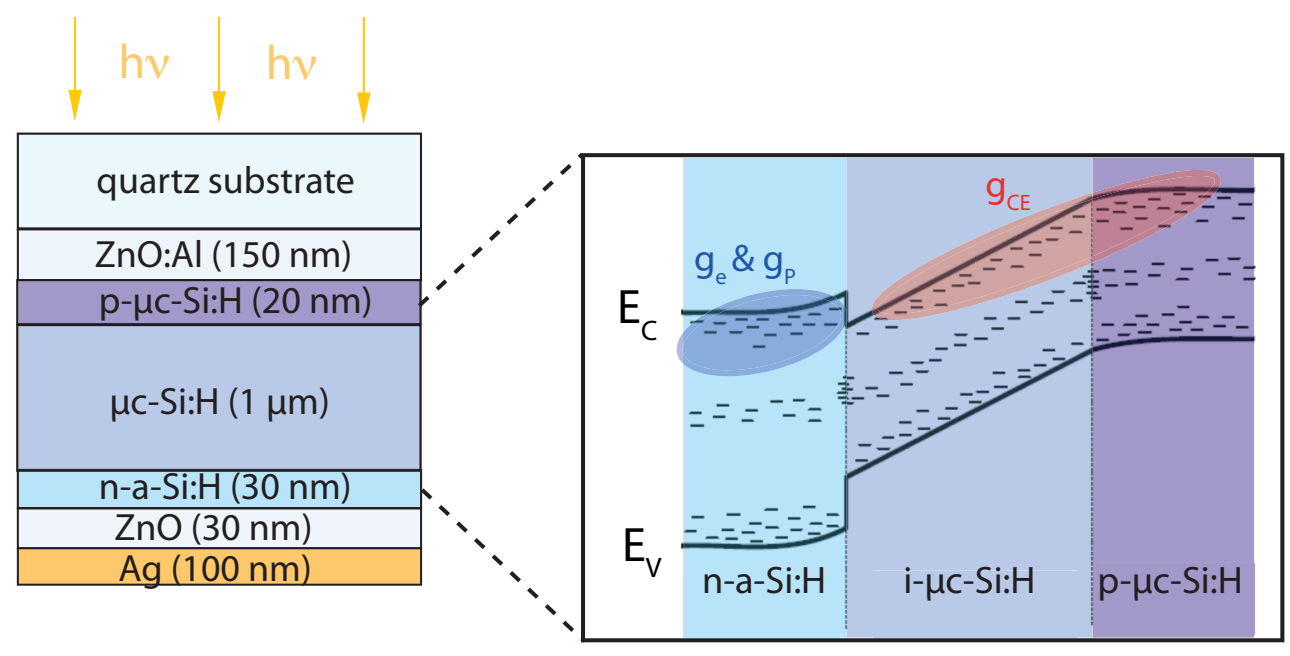

Figure 1: Left: layer structure of the $\mu \mathrm{c}-\mathrm{Si}: \mathrm{H}$ p-i-n thin-film solar cells. Right: schematic representation of the energy-band diagram at room temperature without illumination. The amorphous layer can be identified by the larger band gap. The detected signals are assigned to hopping transport via shallow conduction band tail states at a $g$-value of $g_{\mathrm{CE}}$ in the $\mu \mathrm{c}-\mathrm{Si}: H$ regions and conduction band tail states at $g_{\mathrm{e}}$ as well as phosphorus donor states at $g_{\mathrm{P}}$ in the amorphous layer.

for spin-dependent recombination via db states could be found in the EDMR spectra. Possibly the strong hopping signals superimpose a weaker db recombination signal. This hypothesis is consistent with the results of a previous study, where an EDMR recombination signal could only be found in purely microcrystalline p-i-n solar cells, but not in cells with amorphous p- and/or n-doped layers exhibiting strong EDMR signals from hopping transport via tail states [19].

Despite the fact that detailed insight about spin-dependent transport processes could be obtained from above mentioned studies, several impor- 
tant questions remained unsolved. The limited spectral resolution at X-band frequencies did not allow for extracting field-dependent EDMR line shapes. These parameters reveal important information about the electronic structure of paramagnetic states, contained in the distribution and canonical values of the electronic $g$-matrix. In order to obtain these pieces of information, high field/high frequency EDMR experiments are required. Furthermore, recent X-band pEDMR works on $\mu \mathrm{c}-\mathrm{Si}: \mathrm{H}$ solar cells assumed spin pair processes to be responsible for the observed spin-dependent transport processes. These processes do not depend on the equilibrium Boltzmann spin polarization. However, studies limited to one polarization regime may miss other important spin polarization dependent transport processes like e.g. recombination of electrons and holes in the conduction and valence bands with thermalized spin polarization [24], trapping of conduction electrons at ionized donors [25], spin-dependent scattering [26] or polarization transfer from donors to delocalized electrons [27]. In order to investigate the impact of these processes, EDMR experiments at different resonance fields and/or temperatures are required. Finally, an unambiguous assignment of paramagnetic specimen in $\mu \mathrm{c}-\mathrm{Si}: \mathrm{H}$ solar cells by $\mathrm{X}$-band EDMR is limited by the fact that field-dependent (Zeeman interaction) and field-independent (spin-spin interactions) line shape contributions cannot be discriminated.

In order to lift these restrictions we extend X -band EDMR in the present work to a multi-frequency EDMR approach. Thereby, we benefit from the enhanced spectral resolution at Q- and W-band frequencies and discriminate between field-dependent and field-independent line shape parameters, advantages which are routinely used in conventional EPR spectroscopy [13, 28]. 
With this approach it is possible to assign magnetic field-dependent line broadening contributions to the different spectral components. Furthermore, we will investigate whether significant thermal spin polarization, which exists for low temperature W-band EDMR, influences the nature of the spindependent processes and thereby EDMR spectrum.

After briefly describing the X-, Q- and W-band setups, we present the results of the multi-frequency study. First, the experimental spectra are described followed by the explanation of the methods for the spectral deconvolution. Afterwards we determine precise $g$-values of the e and $\mathrm{P}$ signals and corroborate hyperfine (hf) interaction as the reason for the splitting of the $\mathrm{P}$ resonance and consider the line profile of the $\mathrm{CE}$ line making use of the decomposed spectra. We analyze the influence of field-dependent line broadening effects of the deconvoluted $\mathrm{CE}$ and e signals on the spectral resolution in the EDMR spectra. Finally, we discuss the effect of thermal spin polarization on the EDMR spectrum.

\section{Materials and methods}

\subsection{Principle of EDMR}

The concept of pulsed EDMR $[16,17]$ is explained by discussing the most prominent spin-dependent charge transport processes in the investigated device at low temperature. Illumination of the $\mu \mathrm{c}-\mathrm{Si}: \mathrm{H}$ device excites electrons into the conduction band, which then thermalize into energetically lower localized conduction band tail states. Hopping transitions of carriers into singly occupied paramagnetic tail states are spin-dependent because the charge carriers obey the Pauli exclusion principle such that doubly occupied 
states have to be in an antisymmetric singlet spin configuration. Hence, the transition probability of a carrier into a singly occupied state is determined by the mutual spin orientation $[15,17,29]$. A surplus of pairs with a triplet character blocking the spin-dependent transport occurs when assuming that weakly coupled spin pairs are generated by continuous illumination having all 4 eigenstates $\left(\left|T_{+}\right\rangle\right.$and $\left|T_{-}\right\rangle$with pure triplet character as well as $|2\rangle$ and $|3\rangle$ with triplet and singlet character) with equal probability. In the steady state the high triplet proportion is further increased by the short life time of the singlet pairs due to fast spin-dependent transitions, while triplet pairs are long-lived.

The application of mw pulses allows the manipulation of the relative spin orientation of the spin partners and results in an increase of the singlet content. This leads to a higher hopping transport probability and a higher sample conductivity. The response of the sample conductivity is measured via current-detection in EDMR experiments (Fig. 2). Directly after the pulse a rapid change of current is detected due to the enhanced singlet content. In the course of time, spin relaxation and reoccupation of tail states with electrons by light excitation as well as loss of carriers reestablish the steady state singlet and triplet proportion of the spin pairs, which is observed as an EDMR signal change in the experiment. The EDMR spectra shown in this study result from digital boxcar integrations over time intervals of the current transients (Fig. 2, inset).

\subsection{Sample preparation}

The investigated solar cell (Fig. 1) is a single junction p-i-n (p- $\mu \mathrm{c}-\mathrm{Si}: \mathrm{H}$ / i- $\mu \mathrm{c}-\mathrm{Si}: \mathrm{H} / \mathrm{n}-\mathrm{a}-\mathrm{Si}: \mathrm{H})$ diode prepared in a superstrate configuration with 


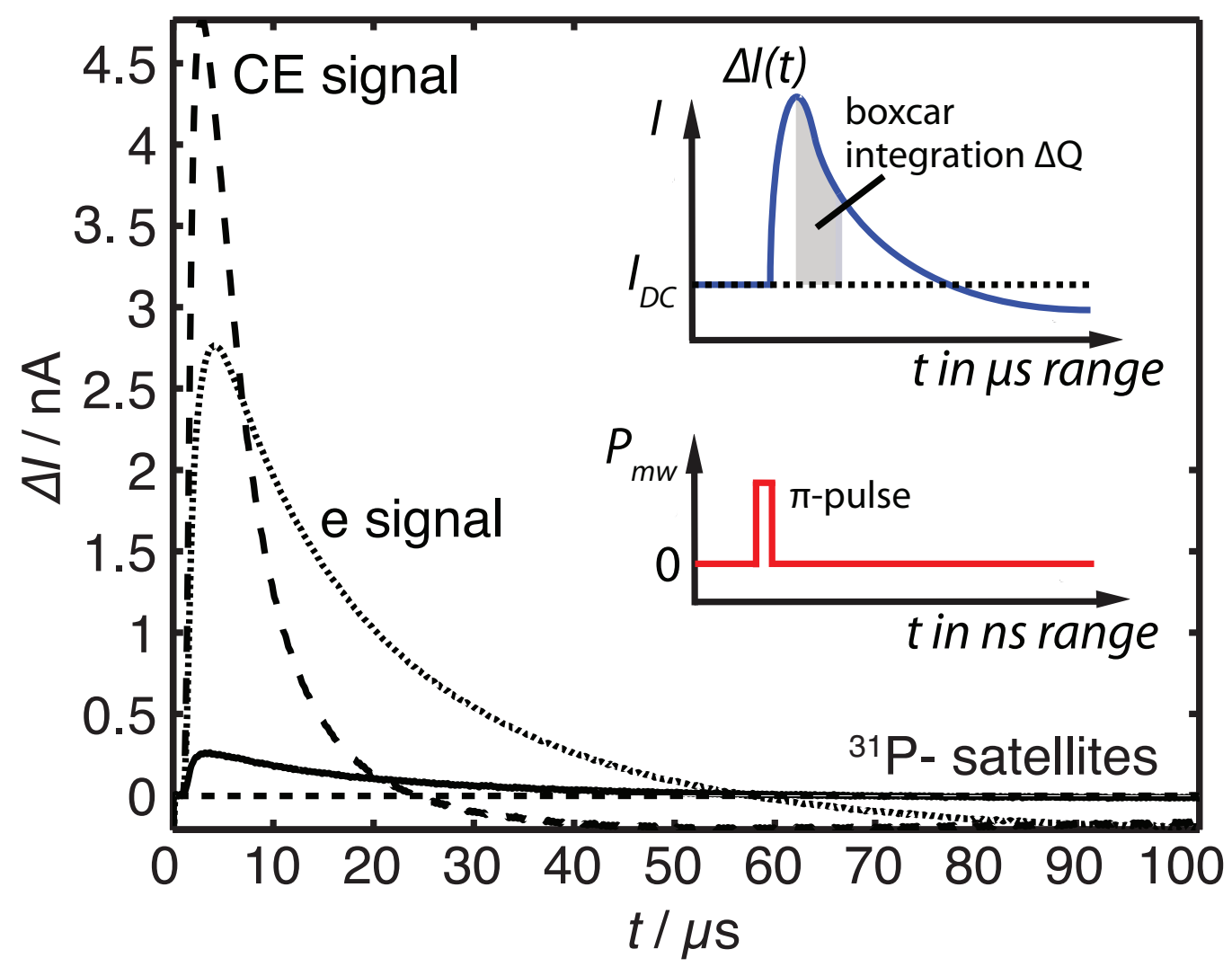

Figure 2: A change of the relative spin orientation by mw pulses leads to a transient current response $\Delta I(t)$. Here the current response of the e, $\mathrm{P}$, and $\mathrm{CE}$ signals from the $\mu \mathrm{c}-$ Si:H solar cells is shown. EDMR field spectra are generated by integrating over a certain time interval of the transient current response (digital boxcar integration). The maximum response is achieved by applying mw $\pi$-pulses.

a process developed for a high quality device [30]. The cell was deposited by plasma enhanced chemical vapor deposition (PECVD) on a glass substrate (Corning 1737) covered with a $\mathrm{ZnO}$ front contact. The crystallinity of the $1 \mu \mathrm{m}$ thick intrinsic $\mu \mathrm{c}-\mathrm{Si}: \mathrm{H}$ absorber layer is approximately $65 \%$ according to Raman measurements [31]. The n-a-Si:H layer was doped with 
phosphorus and the p- $\mu \mathrm{c}-\mathrm{Si}: H$ layer with boron. In order to perform EDMR measurements solar cells of $1 \times 1 \mathrm{~mm}^{2}$ with a special contact configuration were prepared with laser scribing. The preparation sequence and laser scribing procedure was presented in more details elsewhere $[19,32]$. The total size of the sample for the EDMR investigations $\left(55.0 \times 2.9 \times 0.7 \mathrm{~mm}^{3}\right)$ is limited by the cavity size at X- and Q-band.

\subsection{EDMR instrumentation}

We performed pulsed EDMR experiments utilizing Bruker Biospin spectrometers of type Elexsys E580 and E680 working at frequencies of 9.7, 34 and $94 \mathrm{GHz}$ (X-, Q- and W-band). The time-dependent conductivity change was measured after pulsed mw excitation as a function of the static magnetic field $B_{0}$ while the resonance frequency $\nu_{0}$ was kept constant. At every field point current transients of up to $100 \mu$ s were recorded.

The sample was illuminated in all experiments by a stabilized DC driven halogen cold light source $(P=150 \mathrm{~W})$ from Polytec. The sample cooling was realized by CF935 cryostats and ITC503 temperature controllers from Oxford Instruments to perform EDMR experiments at $10 \mathrm{~K}$ to benefit from the high signal-to-noise ratio (SNR). A combined voltage source and current amplifier produced by Elektronik Manufaktur Mahlsdorf was used [19] to detect the spin-dependent change in conductivity. The cell was operated at $1 \mathrm{~V}$ reverse bias in $\mathrm{X}$ - and Q-band to extract the photo-generated charge carriers from the device leading to photocurrents between 4 and $8 \mu \mathrm{A}$ dependent on the illumination conditions. Smaller light intensities due to the setup at W-band reduced the photocurrent at $1 \mathrm{~V}$ bias voltage and $10 \mathrm{~K}$ significantly (see Sec. 2.3.2). Hence we used here a higher voltage of $1.5 \mathrm{~V}(3.2 \mu \mathrm{A})$ and $5 \mathrm{~K}$ to 
further increase the detected spin-dependent response. The change of these parameters did not influence the spectral information but only led to a better SNR.

\subsubsection{Experiments at $X$ - and $Q$-band}

At X- and Q-band frequencies the sample was mounted in a mw resonator as for conventional EPR measurements. A dielectric ring resonator ER 4118X-MD5 was used for X-band experiments and a lab built cavity resonator suitable for samples with maximum diameter up to $3 \mathrm{~mm}$ at Q-band (see table 1). The excitation of photo-carriers in the solar cell was achieved at $9.7 \mathrm{GHz}$ by illumination through the optical window in the resonator. At $34 \mathrm{GHz}$ the light was coupled into an optical fiber guided in the interior of the sample rod to the sample. At the end of the fiber a small rectangular prism of $0.7 \mathrm{~mm}$ edge length was fixed to reflect the radiation directly onto the active area of the device.

EDMR spectra were measured as function of mw power to optimize the signal intensity without affecting the line shape by broadening effects. At $9.7 \mathrm{GHz}$ and $34 \mathrm{GHz} \pi$-pulses of $100 \mathrm{~ns}$ and $70 \mathrm{~ns}$, respectively, where found as optimum conditions.

The magnetic field was calibrated at these two frequencies using a LiLiF standard with a known $g$-value of 2.002293(2) [33].

\subsubsection{Experiment at $W$-band}

At frequencies higher than Q-band an EDMR setup is much more difficult to implement in dielectric or fundamental mode cavity resonators due to the smaller wave lengths. Lang et al. [34] were able to employ a commer- 
cial Bruker Teraflex EN 600-1021H resonator at $94 \mathrm{GHz}$ allowing a sample diameter of $0.87 \mathrm{~mm}$ to investigate silicon field-effect transistors (active area of $160 \times 40 \mu \mathrm{m}^{2}$, sample size $\left.15.0 \times 0.5 \times 0.3 \mathrm{~mm}^{3}\right)$.

This resonant setup could not be used here to investigate the same solar cell already used for the X- and Q-band experiments because the chosen size of the solar cell exceeded the maximum sample dimensions and because illumination of the thin-film solar cell is necessary. For this reason we used a non-resonant setup. The sample is placed underneath a mw transmission line consisting of an oversized rectangular (Q-band) waveguide, which is tapered down to W-band dimensions at the end. This non-resonant structure allows for the insertion of several $\mathrm{mm}^{2}$ large samples and convenient sample illumination. In an EDMR experiment the solar cell is illuminated by visible light (via an optical fiber) and mw through the glass substrate. To increase $B_{1}$ at the sample the back side of it was covered with an additional reflective metallic layer. The maximum available $\mathrm{mw}$ power of $0.4 \mathrm{~W}$ in this non-resonant setup yielded $\pi$-pulses of 500 ns (see table 1 ).

A BDPA (1,3-bisdiphenylene-2-phenylallyl) sample was used for transient nutation experiments to estimate possible $B_{1}$ inhomogeneity in the nonresonant setup. It was deposited as a thin film on a glass substrate with a total film area larger than the active area of the solar cell. Nutations could be detected over several periods indicating small inhomogeneity effects.

The field axis calibration was done at W-band with the help of a small BDPA sample $(g=2.00264$ [35]) fixed on the glass substrate of the solar cell next to the active area. Calibration measurements were performed for mw frequencies between 93.7 and $94.4 \mathrm{GHz}$ to cover a sufficient range of the field 


\begin{tabular}{|c|c|c|c|}
\hline frequency & setup & $\pi$-pulses & B $_{1}$-field amplitude \\
\hline $9.7 \mathrm{GHz}$ & ER 4118X-MD5 & $100 \mathrm{~ns}$ & $178 \pm 12 \mu T$ \\
\hline $34 \mathrm{GHz}$ & lab built resonator & $70 \mathrm{~ns}$ & $255 \pm 24 \mu \mathrm{T}$ \\
\hline $94 \mathrm{GHz}$ & non-resonant setup & $500 \mathrm{~ns}$ & $36 \pm 8 \mu T$ \\
\hline
\end{tabular}

Table 1: Overview of characteristic quantities of the different frequency bands. The $\pi$ pulse lengths and $B_{1}$-field amplitudes $\left(B_{1}=\frac{\Omega_{R a b i}}{\gamma}\right)$ were determined by EDMR transient nutation experiments [36].

axis.

\section{Results and discussion}

The EDMR spectra of the $\mu \mathrm{c}-\mathrm{Si}: \mathrm{H}$ solar cell at all three measurement frequencies are shown in Fig. 3. The center of the current integration windows was chosen such that the maxima at the two field positions indicated by vertical lines have equal intensity to show the differences in spectral resolution. At X- and Q-band an integration window of $100 \mathrm{~ns}$ in the transients recorded was used. Due to the reduced SNR of the transients at W-band an integration window of $7.45 \mu$ s width was chosen in order to achieve a sufficient SNR.

The two main signal contributions are assigned to the CE resonance at $g \approx 1.998$ (dashed line) and the e resonance at $g \approx 2.005$ (dotted line). The $\mathrm{P}$ signal pair is not well resolved in these spectra as it is outside of the covered field range in X-band or buried under the stronger resonances at W-band. Only in the Q-band spectrum it can be observed as broad tails of the central lines. 


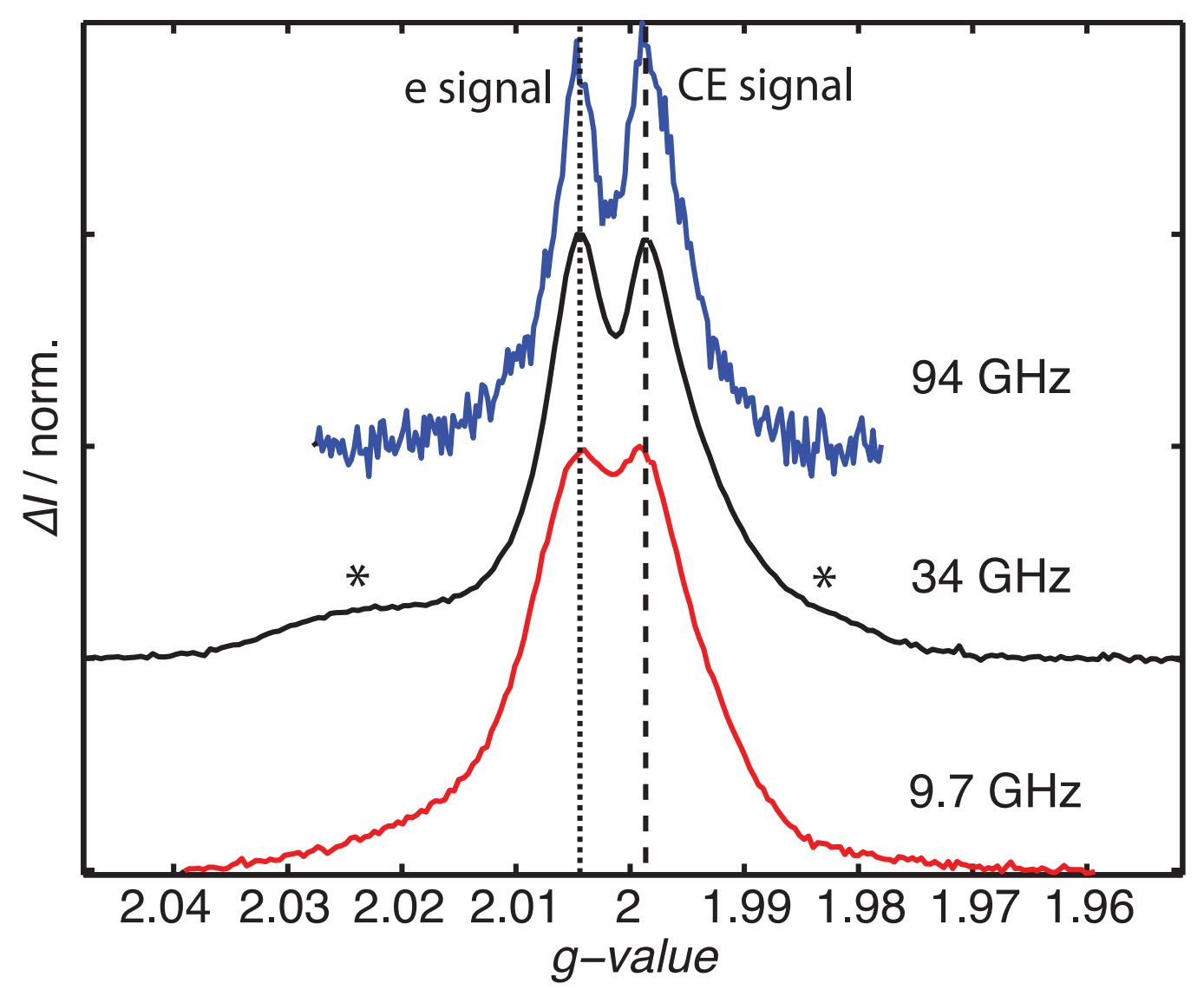

Figure 3: EDMR spectra of the $\mu \mathrm{c}-\mathrm{Si}: \mathrm{H}$ solar cell at $9.7,34$ and $94 \mathrm{GHz}$. The narrow signals are assigned to CE states $(g \approx 1.998$, dashed line) and e states $(g \approx 2.005$, dotted line). At $34 \mathrm{GHz}$ the broad hf satellites of phosphorus donors centered around $g \approx 2.004$ can be observed as broad shoulders (marked with asterisks).

\subsection{Spectral deconvolution}

The basis for the analysis of the EDMR spectra at different frequencies and for the determination of field-dependent and field-independent line broadening effects is the knowledge of the line shapes and widths of the different signals. For the determination of these line parameters the X-band 
EDMR spectrum of the $\mu \mathrm{c}-\mathrm{Si}: \mathrm{H}$ solar cell was fitted with symmetric line profiles $[18,19]$. The CE and e signal were described by Lorentzian line shapes, the $\mathrm{P}$ satellites were approximated by a pair of Gaussian lines. These line shapes, especially the Lorentzian profiles, were chosen because they adequately fit the EDMR signals. However, we note that the Lorentzian line shape should not be interpreted as a result of homogeneous broadening. In a first step, the same fitting approach was applied to the X-, Q- and W-band data of our study as demonstrated examplarily in Fig. 4 a) for the Q-band data set. The fit yields the following results: The EDMR spectrum can be well described in the low field range up to $\approx 1245 \mathrm{mT}$ by Lorentzian lines for the $\mathrm{CE}$ and e signals, respectively and one Gaussian line for the low-field $\mathrm{P}$ signal. At the high field side of the CE signal a significant deviation between experimental spectrum and fit is observed (marked in shaded red). This deviation can be reduced neither by changing the CE line position nor its linewidth. The high field side of this resonance is significantly broader than the low field side. This asymmetric CE line profile is found in the EDMR spectra at all three frequency bands.

The fitting procedure based on symmetric fit functions is not sufficient to describe the spectrum consisting of partly asymmetric lines and to extract reliable parameters. Hence in a second step we use the time dependence of the pulsed EDMR signal transients to decompose the strongly overlapping signal contributions. Our approach is again demonstrated utilizing the Qband data set and applied then also to the X- and W-band data. 


\subsubsection{Decay-associated spectra}

The time dependence of the Q-band data set is shown in Fig. $4 \mathrm{~b}$ ). Spectra extracted at different times after the mw-pulse by integrating over a time interval of $100 \mathrm{~ns}$ in the current transient are displayed. Starting from the maximum of current change at $3.7 \mu$ s after the mw-pulse the EDMR signal intensity decreases, changes sign and decays to zero for long times after the mw-pulse. This signal evolution is connected with different time constants over the spectrum: in the field range of the CE signal values of 4-5 $\mu \mathrm{s}$ and 14-16 $\mu \mathrm{s}$ are found, while in the range of the e and $\mathrm{P}$ resonances time constants of 8-9 $\mu$ s and 24-27 $\mu$ s dominate. Similar time constants for the e and $\mathrm{P}$ resonances are expected as these paramagnetic centers contribute to the same transport path [19]. The difference in the time behavior is used to separate the spectra into so-called decay-associated spectra (DAS) [37]. The EDMR spectrum is decomposed by approximating the current transients with the help of a fit function consisting of four exponential functions with time constants restricted to the above mentioned time intervals. Plotting the prefactors of the exponential functions connected with the four time constants in dependence on the magnetic field results in the DAS. Fig. $4 \mathrm{c}$ ) shows the DAS of the Q-Band data set. A clear separation regarding the e and P (shaded in grey), as well as the CE signal is seen. Nevertheless, the high-field satellite of the $\mathrm{P}$ pair overlapping with the strong $\mathrm{CE}$ line is not reproduced between 1245 and $1460 \mathrm{mT}$ with the expected Gaussian line fitting well the low-field satellite. The reason is the high intensity of the CE line in comparison to the $\mathrm{P}$ signal in this field range as well as the relatively small difference in the time constants characterizing the different resonances. 
Hence we make use of further decomposition methods.

\subsubsection{Deconvolution utilizing signal dynamics}

As seen in the time dependence of the EDMR spectrum in Fig. 4 b), the CE signal decays faster and changes sign at roughly $t \approx 25 \mu \mathrm{s}$. At this time this resonance does not contribute to the spectrum. Only the e and $\mathrm{P}$ signals show a contribution as they decay with larger time constants. Their contribution to the spectrum disappears at around $t \approx 57 \mu$ s. Here only the CE signal (with inverted sign) is observed.

To precisely determine the time points of zero crossing of the e, $\mathrm{P}$ and $\mathrm{CE}$ lines for a detailed spectral analysis, the time behavior of the single contributions is calculated. This is achieved by fitting the Q-band EDMR spectrum at $t=7.7 \mu \mathrm{s}$ in the low field range upto $1247 \mathrm{mT}$ (near the maximum of the CE line) by three symmetric lines yielding a very good representation of the experimental spectrum in this field range (Fig. 4 a)). This fit is repeated for all time steps in the signal transients keeping the linewidths and positions of all signals constant and solely fitting the varying amplitudes. The time dependent change of the line amplitudes as well as the zero intersection time points are obtained by this procedure.

Hence we are able to extract the individual signals from the X- and Qband spectra with this method. A disadvantages of the approach is the low signal quality especially of the $\mathrm{CE}$ line as the SNR at $t \approx 57 \mu \mathrm{s}$ is already clearly reduced. Application of this method to W-band data yields decomposed spectra of low SNR. Hence an improved approach is suggested in the next section. 


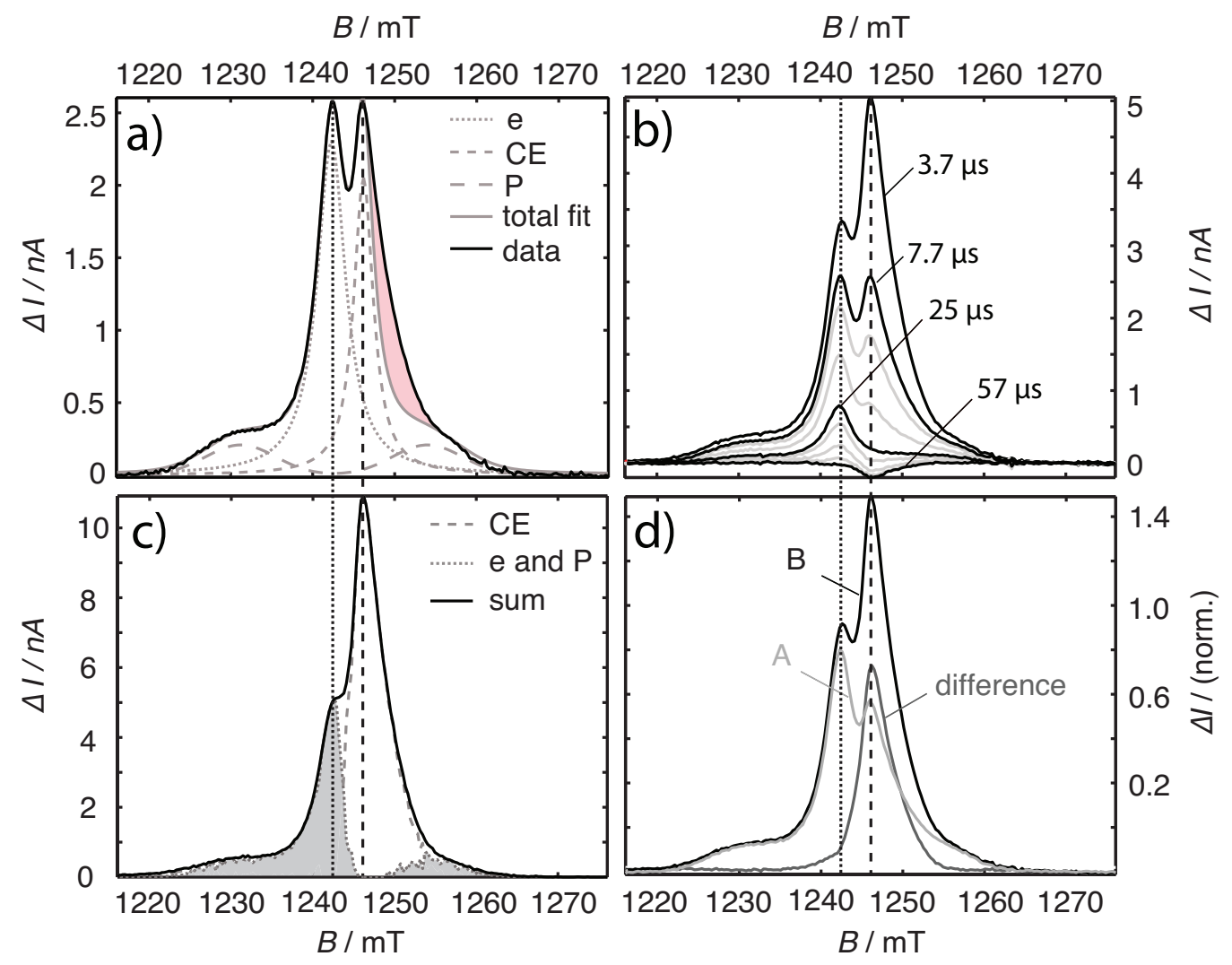

Figure 4: Deconvolution of the EDMR spectra demonstrated for the Q-band data set. a) shows the spectrum at $t=7.7 \mu$ s fitted by symmetric lines. On the high field side deviations between fit and spectrum are observed (marked in shaded red). b) shows the time dependence of the spectrum. At $t=7.7 \mu \mathrm{s}$ the e and CE signals exhibit equal amplitudes. At $t \approx 25 \mu$ s only the e and $\mathrm{P}$ signals contribute. At $t \approx 57 \mu$ s solely the $\mathrm{CE}$ line is observed. c) shows the deconvolution into decay-associated spectra. The sum of the prefactors of the two exponential functions fitting the transients of the $\mathrm{CE}$ line (dashed line) on the one hand, and the transients of the e and P signals (dotted line, area shaded in grey) on the other hand are shown in dependence on the magnetic field. d) shows the decomposition of the spectrum by subtracting spectra A and B of different relative signal intensities. For details see Sec. 3.1.3. 


\subsubsection{Deconvolution utilizing relative signal intensities}

In order to improve the spectral deconvolution of the overlapping signal contributions we employed an additional approach making use of the different signal dynamics in an alternative way. The e and $\mathrm{P}$ signals have indistinguishable dynamics, which clearly deviate from CE signal dynamics. This leads on the one hand to different relative amplitudes of the CE signal compared to the e and $\mathrm{P}$ signals at different times and, on the other hand, to constant relative intensities of the e and $\mathrm{P}$ signals. The two previous deconvolution methods also clearly show that the low field part of the EDMR spectra below $1235 \mathrm{mT}$ is exclusively due to the e and $\mathrm{P}$ resonances. Thus, a scaling of two spectra extracted at different times after the mw pulse to identical intensity of the low-field $\mathrm{P}$ resonance immediately results also in identical intensity for the e resonance. This is shown for the Q-band data set in Fig. $4 \mathrm{~d}$ ), where two spectra $\mathrm{A}$ and $\mathrm{B}$ with $\mathrm{A}$ integrated over the time interval between $3.7 \mu \mathrm{s}$ and $29.9 \mu \mathrm{s}$ and B between $3.65 \mu \mathrm{s}$ and 3.75

$\mu$ s are scaled to identical intensity in the low-field part. Subtraction of the spectra $\mathrm{A}$ and $\mathrm{B}$ removes the signal contributions of the $\mathrm{P}$ and e resonances and exclusively retains the $\mathrm{CE}$ resonance. The results for the $\mathrm{CE}$ resonance are in agreement with the previous results of the deconvolution methods, but show a significantly improved SNR, in particular in the case of the W-band data set.

The final decomposed spectra for the e and $\mathrm{P}$ signals and the CE signal at all three frequency bands are shown in Fig. 5 a) to c). The magnetic parameters that can be extracted for the individual signal contributions will be discussed in the next section starting with the e and $\mathrm{P}$ resonances. 


\subsection{Analysis of the $e$ and $P$ resonances}

The extracted e and $\mathrm{P}$ signals from the a-Si:H layer of the solar cell are given in Fig. 5 a). Fitting the spectra with symmetric Lorentzian lines for the e signal and two broad Gaussian lines for the $\mathrm{P}$ signal yields very good results (Fig. 5 b), table 2). The obtained $g$-values are $g_{\mathrm{e}}=2.0050(3)$ and $g_{\mathrm{P}} \approx 2.005(2)$ at X-band. At Q-band we get $g_{\mathrm{e}}=2.0047(2)$ and $g_{\mathrm{P}}=2.0041(5)$. The precision of the results is increased in this frequency band due to a high SNR of the spectrum and the larger Zeeman splitting. No further improvement of the $g_{\mathrm{e}}$ precision was achieved at W-band so far, as the SNR of the W-band spectrum is limited due to the small $B_{1}$ field strengths in the non-resonant setup. For the e resonance we obtain $g_{\mathrm{e}}=2.0047(2)$. An asymmetry of the e signal cannot be resolved even at W-band. The P signal shows a low SNR at $94 \mathrm{GHz}$ due to the weak intensity, which hampers the determination of the $g$-value. Using the results of the other frequency bands, the $\mathrm{P}$ signal pair at $\mathrm{W}$-band is well described, yielding $g_{\mathrm{P}}=2.004(1)$. The linewidth has a FWHM of $\Delta B_{\mathrm{P}} \approx 12.4 \mathrm{mT}$ at $\mathrm{X}$ - and Q-band and 12(2) $\mathrm{mT}$ at W-band. The signal splitting of $a_{0}=21.2(6) \mathrm{mT}$ at $\mathrm{X}$ - and Q-Band, as well as $a_{0}=21(3) \mathrm{mT}$ at $\mathrm{W}$-band is unchanged within errors at all measured frequencies. These obtained results of the $\mathrm{P}$ signal position, width and splitting are consistent for all three frequency bands with respect to the interpretation as hf satellites.

\subsection{Asymmetry of the CE line}

The CE resonance from the microcrystalline part of the sample could be extracted reliably from the EDMR spectra with the help of its dynamics despite the strong overlap with the e signal and the high field P satellite (Fig. 


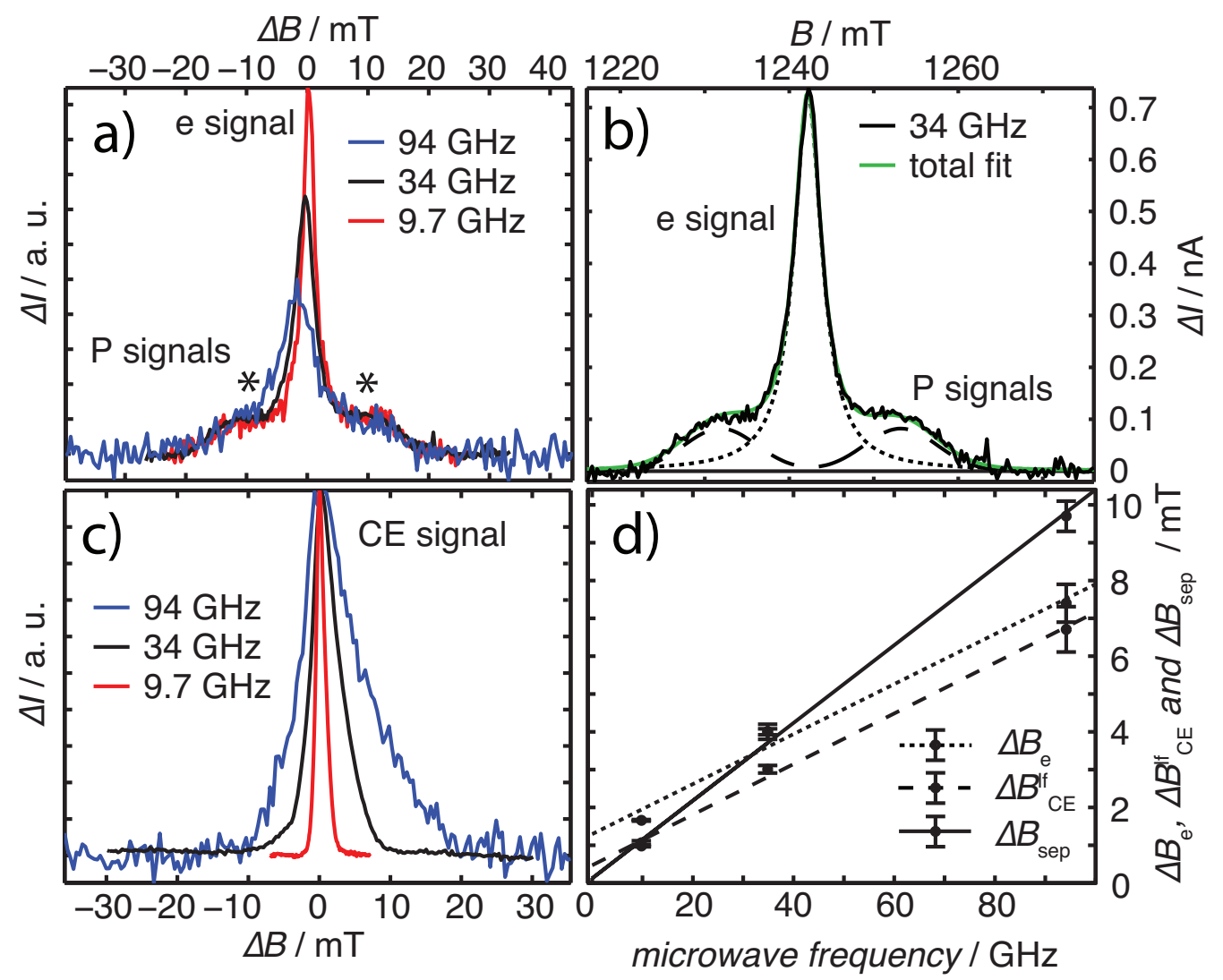

Figure 5: Spectral decomposition results achieved by methods described in Sec. 3.1. a) Narrow e signal (central line), together with P signals (marked with asterisks), extracted from signal dynamics (Sec. 3.1.2). Spectra are shifted in field relative to the center of the $\mathrm{P}$ doublet and normalized to $\mathrm{P}$ signal maxima. b) Q-band spectrum recorded at $25 \mu \mathrm{s}$, together with spectral fits to the e and $\mathrm{P}$ resonances (dotted and dashed lines, respectively). c) Deconvoluted CE resonances obtained from relative signal intensities (Sec. 3.1.3). Spectra are shifted in field relative to the center of the CE line and normalized to the signal maxima. d) $\mathrm{CE}$ and e signal linewidths (FWHM). $\Delta B_{\mathrm{CE}}^{\mathrm{lf}}$ and $\Delta B_{\mathrm{e}}$ and the spectral separation of both resonance lines $\Delta B_{\text {sep }}$ plotted against EDMR resonance frequencies. Dashed, dotted and solid lines represent fits to the respective parameters. 


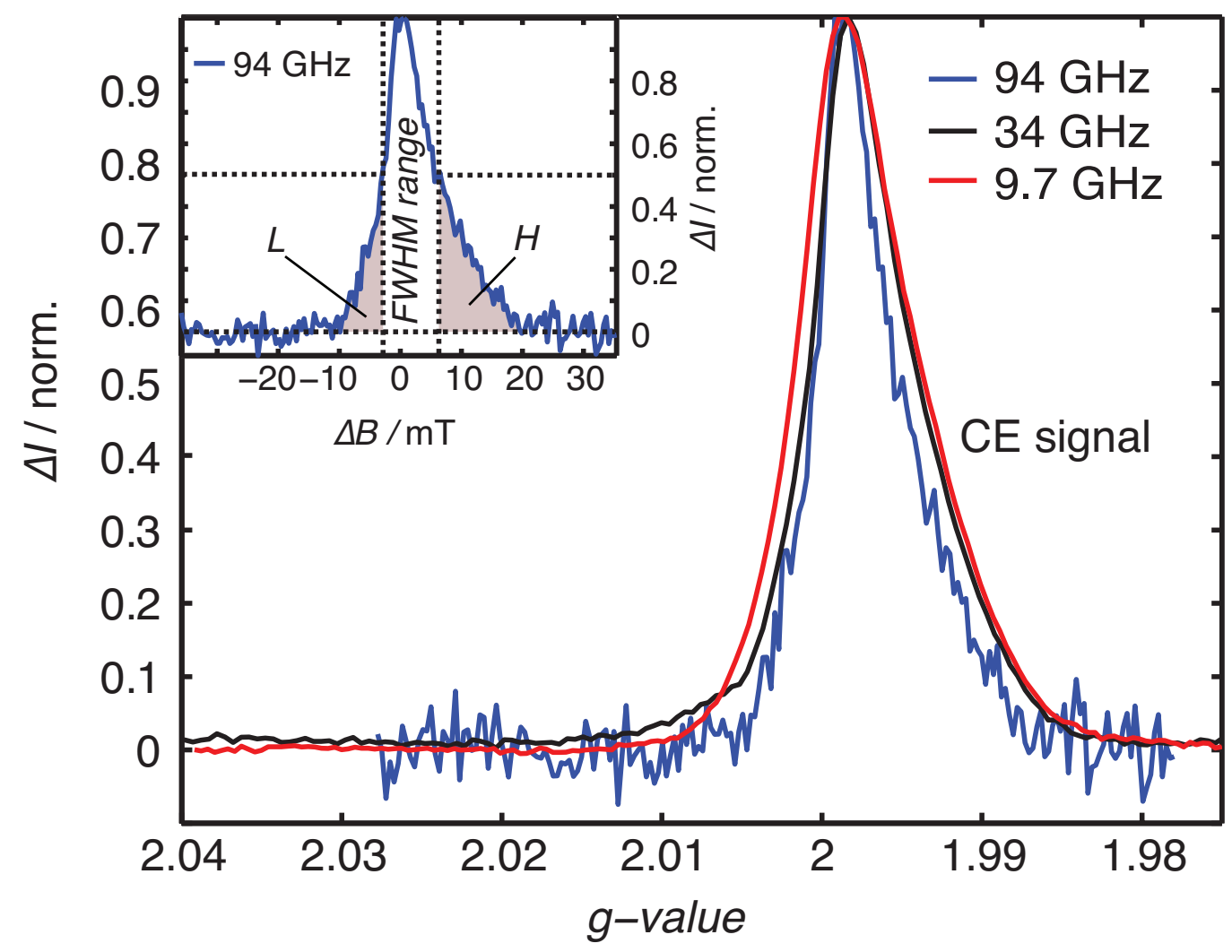

Figure 6: The extracted $\mathrm{CE}$ line at all three frequency bands is displayed on the g-value axis. In this picture the increased resolution of the asymmetry is seen. Inset: The degree of asymmetry of the CE profile (W-band spectrum) is determined by comparing the spectral contribution from the field intervals above (interval $H$ ) and below (interval $L$ ) the FWHM range of the line. 


\begin{tabular}{|c||c||c||c|c|c|}
\hline frequency & $g_{\mathrm{CE}}$ & $g_{\mathrm{e}}$ & $g_{\mathrm{P}}$ & $\Delta B_{\mathrm{P}}$ & $a_{0}$ \\
\hline X-band & $1.9988(3)$ & $2.0050(3)$ & $2.005(2)$ & $12.4(6) \mathrm{mT}$ & $21.2(6) \mathrm{mT}$ \\
\hline Q-band & $1.9984(2)$ & $2.0047(2)$ & $2.0041(5)$ & $12.4(4) \mathrm{mT}$ & $21.2(6) \mathrm{mT}$ \\
\hline W-band & $1.9988(2)$ & $2.0047(2)$ & $2.004(1)$ & $12(2) \mathrm{mT}$ & $21(3) \mathrm{mT}$ \\
\hline
\end{tabular}

Table 2: Results of the evaluation of the $\mathrm{CE}$, e and $\mathrm{P}$ signals for all measured frequency bands. The $g$-values $g_{\mathrm{CE}}$ ( $g$-value of line maximum), $g_{\mathrm{e}}$ and $g_{\mathrm{P}}$ as well as the $\mathrm{P}$ doublet linewidth $\Delta B_{\mathrm{P}}(\mathrm{FWHM})$ and its splitting $a_{0}$ are given.

3). It reveals an asymmetric profile (Fig. 6), which is observed in all three frequency bands. The resonance with its signal maximum at $g=1.9986(3)$ reaches $g$-values up to $g \approx 2.004$ on the low field side and down to $g \approx 1.988$ on the high field side. The integrated spectral intensities of the CE profile in the field ranges above (interval $H$ ) and below (interval $L$ ) the FWHM range of the line were compared to quantify the degree of asymmetry (Fig. 6, inset). The ratio $r=\frac{H}{L}$ yields 1.2, 1.3 and 1.8 for X-, Q- and W-band, respectively. The values clearly deviating from 1.0 indicate an asymmetric character at all measured frequencies, with the largest asymmetry seen at W-band.

The CE resonance is interpreted in the literature as originating from the crystalline region of the $\mu \mathrm{c}-\mathrm{Si}: \mathrm{H}$ material. At first the $\mathrm{CE}$ states were assigned to free carriers in the conduction band of the crystalline grains [38-40]. Later on the states were attributed to shallow conduction band tail states in the $\mathrm{Si}$ crystallites acting as electron traps [41, 42]. However, the microscopic center associated with the CE resonance could not be unambiguously identified yet.

The asymmetric line profile with field-dependent high- and low-field line- 
widths clearly shows a Zeeman interaction dominated line-broadening effect for the paramagnetic states giving rise to the $\mathrm{CE}$ resonance. It is important to note, however, that the particular line shape cannot be reconciled with an axial $g$-matrix under the assumption of an isotropic powder sample. Instead, it has been regarded before as being due to a superposition of two lines [43]. However, the magnetic parameters suggested in [43] do not fit our data set.

An asymmetric line profile can result from a non-isotropic distribution of paramagnetic centers. Possible candidates for these centers are dangling bonds at (internal) interfaces between amorphous and crystalline regions in the $\mu \mathrm{c}$-Si:H layer. Given the fact that the crystalline regions exhibit a preferential orientation with respect to the substrate, the resulting restricted powder pattern will lead to an asymmetric line shape that differs from a full powder pattern expected for a purely isotropic distribution. However, a recent EDMR study on recombination in a-Si:H/c-Si heterostructures, which can be regarded as model systems for microcrystalline $\mathrm{Si}$, has revealed an orientation dependent $g$-matrix with main values of $g_{\perp}=2.008(1)$ and $g_{\|}=2.001(1)$ for the paramagnetic centers located at the interface [44]. This $g$-value range clearly deviates from the $g$-value of the CE center. In consequence, an anisotropic distribution of paramagnetic states at internal a-Si:H/c-Si interfaces cannot be responsible for the CE line asymmetry.

Therefore, we suggest a different origin for the line shape, still assuming the $\mathrm{CE}$ resonance to arise from the crystalline part of the $\mu \mathrm{c}-\mathrm{Si}: \mathrm{H}$ material as inferred from previous EPR/EDMR investigations [23, 39, 45].

$\mu \mathrm{c}-\mathrm{Si}: \mathrm{H}$ contains small crystallites separated by amorphous material. These crystallites can be regarded as an ensemble of quantum structures. We as- 
sume that the $g$-value of a CE center located inside a Si nanocrystal depends on the respective crystal diameter. Following this idea, the CE line shape is governed by the size distribution of the Si nanocrystals. In the following, we will evaluate whether the geometrical confinement due to the crystal sizes found in $\mu \mathrm{c}-\mathrm{Si}: \mathrm{H}$ may influence the $\mathrm{CE} g$-value and thus provide a credible explanation for the $\mathrm{CE}$ line shape.

$\mu \mathrm{c}-\mathrm{Si}: \mathrm{H}$ deposited under similar conditions as the investigated sample contains crystallites with an average size between 7 and $14 \mathrm{~nm}[31,46]$. While it is impossible to prepare $\mu \mathrm{c}-\mathrm{Si}: \mathrm{H}$ comprising only one particle size and to study the influence of the crystal size on the CE $g$-value directly, data is available on size-selected Si nanoparticles.

Ye et al. [47] studied resonant electron tunneling between c-Si nanocrystals of $\approx 10 \mathrm{~nm}$ diameter embedded in an amorphous $\mathrm{SiO}_{2}$ matrix. They observed several aspects of quantum confinement. Electrons forming delocalized states in bulk semiconductor crystals that are invisible to most EPR techniques, may give rise to strong EPR signals when confined in nanostructures with reduced dimensionality. Nestle et al. [48] for example reported about electrons confined in $\mathrm{Si} / \mathrm{Si}_{1-y} \mathrm{C}_{y} / \mathrm{Si}$ quantum wells having a depth of 50 meV and well width of $6 \mathrm{~nm}$. These paramagnetic centers were detected with EPR signals showing properties (e.g. $g$-value, temperature dependences) resembling those of CE centers [11]. Going to nanostuctures of varying size, Pereira et al. [49] found a clear change of the $\mathrm{P}$ hf splitting of donors in phosphorus-doped c-Si nanocrystals in dependence of the particle size up to diameters $>10 \mathrm{~nm}$ due to dielectric confinement. This is a consequence of the modified donor wavefunction localization. 
Since the crystallite sizes in $\mu \mathrm{c}-\mathrm{Si}: \mathrm{H}$ are in the range of the above mentioned quantum structures, it is reasonable to assume an influence of confinement effects on the energy landscape of the structure and wavefunction localization of the CE centers. A modification of the electron wavefunction leads to a change of the spin orbital coupling, which has a direct influence on the $g$-value of the $\mathrm{CE}$ center. Hence we assume a dependence of the $\mathrm{CE}$ $g$-value on the crystalline grain size at least for a part of the grains. The line shape of the $\mathrm{CE}$ resonance then reflects the grain size distribution within the $\mu \mathrm{c}$-Si:H material and not a powder pattern for a rhombic or axial $g$-matrix.

While we believe that a dependence of the $\mathrm{CE} g$-value on the crystallite size seems plausible, we cannot quantify this effect yet. However, DFT techniques capable of calculating EPR parameters for localized states in thin-film silicon became available recently $[44,50,51]$ and may be employed to study the $g$-value as function of the crystallite diameter.

\subsection{Field-dependence of the spectral resolution}

At $9.7 \mathrm{GHz}$ the e and $\mathrm{CE}$ resonances overlap strongly (Fig. 3). At 34 and $94 \mathrm{GHz}$ the increased Zeeman splitting of the spin states results in a larger separation of both signals on the field axis, which is seen in the central part of the spectra. For a detailed evaluation of the gain in spectral resolution, the linewidth of the decomposed resonances in dependence on the mw-frequency was analyzed (Fig. 5 a) and c)). Therefore, the e linewidth $\Delta B_{\mathrm{e}}$ at the three frequency bands was taken from the fit results of the decomposed e and $\mathrm{P}$ spectra (Fig. $5 \mathrm{a}$ ) and b)). As the CE profile is asymmetric, only

the linewidth $\Delta B_{\mathrm{CE}}^{\mathrm{lf}}$ describing the low field side, relevant for the spectral resolution, is used. 
In Fig. $5 \mathrm{~d}$ ) the separation of the peak positions of the e and CE lines in field $\Delta B_{\text {sep }}$, deduced from the X-, Q- and W-band measurements are plotted as a function of the mw frequency. $\Delta B_{\text {sep }}$ can well be fitted by a straight line as the $g$-values obtained for both species agree within error margins for all three frequency bands. The slope of the linear fit $\Delta g_{\text {slope }}=0.0057(5)$ is in agreement with the average $g$-value difference $\Delta g_{\mathrm{sep}}=g_{\mathrm{e}}-g_{\mathrm{CE}}=0.0061(5)$ calculated from the $g$-values obtained at the three frequency bands.

Fig. $5 \mathrm{~d}$ ) also shows the linewidth change of $\Delta B_{\mathrm{CE}}^{\mathrm{lf}}$ and $\Delta B_{\mathrm{e}}$ in dependence of the mw frequency. The linewidth increases significantly with increasing observation frequency. The three measurements covering one order of magnitude in frequency can be fitted well by a linear function (dotted line for $\Delta B_{\mathrm{e}}$, dashed line for $\left.\Delta B_{\mathrm{CE}}^{\mathrm{lf}}\right)$. This clearly demonstrates that the linewidth is dominated by field-dependent broadening. Two possible origins for field-dependent line broadening are i) $g$-anisotropy and ii) $g$-strain (site-tosite variations of the principal $g$-values, [52]). Investigations of paramagnetic centers in disordered silicon such as the dangling bond defect in amorphous silicon $[10,13]$ show that $g$-anisotropy and $g$-strain dominate the resonance spectrum at Q-band and higher frequencies whereas hf interactions with ${ }^{29} \mathrm{Si}$ and ${ }^{1} \mathrm{H}$ are important at $\mathrm{X}$-band and below.

Hence we consider the development of linewidths in the X-, Q- and Wband spectra to deduce information about the field-dependent linewidth contribution $\Delta g$. A distinction between the two field-dependent broadening effects - $g$-anisotropy and $g$-strain - is impossible. Field-independent contributions, e.g. hf couplings, are neglected at the frequencies used here.

A rough value for $\Delta g$ is obtained from the dependence of the linewidth 
on the resonance frequency as the slope $s_{\mathrm{i}}$ of the linewidth development is determined by:

$$
s_{\mathrm{i}}=\frac{h}{\beta_{\mathrm{e}}} \frac{\Delta g_{\mathrm{i}}}{g_{\mathrm{i}}^{2}} \nu_{0}
$$

[53] with the Planck constant $h$ and the Bohr magneton $\beta_{\mathrm{e}}$. On the basis of this relation $\Delta g_{\mathrm{e}}=0.004(1)$ and $\Delta g_{\mathrm{CE}}^{\mathrm{lf}}=0.0037(6)$ can be calculated for the e and CE resonances. These field-dependent contributions to the $\mathrm{CE}$ and e linewidths are smaller than the $g$-value separation $\Delta g_{\text {sep }} \approx 0.006$ of both resonances. This results in an increased spectral resolution at higher fields and frequencies which makes multi-frequency and especially high-frequency EDMR a powerful tool to obtain information about $g$-values, -anisotropy and -strain of paramagnetic centers in semiconductor devices.

\subsection{Influence of spin polarization}

The EDMR signals reported throughout this article are assumed to originate from spin-dependent transitions between two charge carriers where the symmetry of the spin pair governs the transition rate as described in Sec. 2.1. The signal resulting from such a pair process does not depend on the (thermal) polarization of the spins, i.e., the EDMR spectrum is supposed to be independent of temperature and $B_{0}[29]$. This is consistent with the X-, Q- and W-band spectra shown in Fig. 3, indicating that the same transport process is responsible for the EDMR signals for all frequency bands, although the spin polarization $p=\Delta N / N$ (the ratio between the difference $\Delta N$ in spin down concentration, $N_{\downarrow}$, and spin up concentration, $N_{\uparrow}$, and the total spin concentration $N=N_{\downarrow}+N_{\uparrow}$ ) changes considerably from $2.3 \%$ at X-band $(9.7 \mathrm{GHz}, 10 \mathrm{~K})$ to $42 \%$ at W-band (94 GHz, $5 \mathrm{~K})$. In particular, 
the dynamics of the signals attributed to e, $\mathrm{P}$ and $\mathrm{CE}$ states as well as the relative signal intensities remain nearly constant upon variation of the resonance frequency, whereas the resonance line shapes exhibit a pronounced field-dependence as shown in Fig. 5 c). Drastic changes of the EDMR spectra upon variation of $p$ were observed e.g. for spin-dependent processes at the $\mathrm{Si} / \mathrm{SiO}_{2}$ interface [54]. Here, the authors detected a sign inversion of high-field EDMR signals associated with spin-dependent processes involving

${ }^{31} \mathrm{P}$ donor states near the $\mathrm{Si} / \mathrm{SiO}_{2}$ interface when the temperature was varied between 3 and $12 \mathrm{~K}$. This indicates a $p$-dependent process. In contrast, no evidence for the existence of $p$-dependent processes in $\mu \mathrm{c}-\mathrm{Si}: \mathrm{H}$ solar cells could be found under the experimental conditions used in the present study. This indicates that the hopping processes involving e, $\mathrm{P}$ and $\mathrm{CE}$ states reported in this article are surprisingly robust against changes in the thermal spin polarization over a wide range.

\section{Summary}

In this study light-induced EDMR signals of a single p-i-n $\mu \mathrm{c}-\mathrm{Si}: \mathrm{H}$ solar cell at mw frequencies between 9.7 and $94 \mathrm{GHz}$ were investigated. With the help of deconvolution methods the different spectral components were separated and analyzed in detail.

The $g$-value of the e resonance could be determined precisely with Qand W-band measurements. A signal asymmetry is not resolved even at $\mathrm{W}$-band. The $\mathrm{P}$ signal pair splitting of $\approx 21 \mathrm{mT}$ is constant at all measured frequencies, corroborating the reason for the signal doublet to be hf interaction. The CE line profile shows an asymmetry at all three frequency 
bands, which is most pronounced at W-band. This particular line shape leads us to the suggestion that a size distribution of crystallites accommodating light-activated CE centers is responsible for a $g$-value distribution and hence for the CE resonance asymmetry. From the linewidth development of the e and $\mathrm{CE}$ lines in dependence on the mw frequency a field-dependent linewidth contribution of $\Delta g_{\mathrm{e}} \approx \Delta g_{\mathrm{CE}}^{\mathrm{lf}}=0.004(1)$ could be deduced. The signal splitting of both resonances $\Delta g_{\text {sep }} \approx 0.006$ is larger than the extracted field-dependent broadening, which results in a gain in resolution of EDMR spectra measured at high fields and frequencies. The fact that the EDMR spectra are almost independent of the resonance frequency indicates that the same spin polarization-independent transport process is responsible for the EDMR signals observed in X-, Q- and W-band.

Multi-frequency and especially high-frequency EDMR are thus important tools to characterize paramagnetic centers involved in transport paths in fully processed semiconductor devices. The precise magnetic parameters obtained can further be the fundament for structural identification of these paramagnetic centers by theoretical modeling.

\section{Acknowledgement}

We are grateful to M. Fehr and K.-P. Dinse for helpful discussions. Financial support from BMBF (EPR-Solar network project 03SF0328) is acknowledged.

[1] J. Meier, R. Flückiger, H. Keppner, A. Shah, Complete Microcrystalline P-I-N Solar-Cell - Crystalline or Amorphous Cell Behavior, Appl. Phys. Lett. 65 (7) (1994) 860-862. 
[2] A. Shah, P. Torres, R. Tscharner, N. Wyrsch, H. Keppner, Photovoltaic technology: The case for thin-film solar cells, Science 285 (5428) (1999) 692-698.

[3] O. Vetterl, F. Finger, R. Carius, P. Hapke, L. Houben, O. Kluth, A. Lambertz, A. Muck, B. Rech, H. Wagner, Intrinsic microcrystalline silicon: A new material for photovoltaics, Sol. Energy Mat. Sol. Cells $62(1-2)(2000)$ 97-108.

[4] Y. Mai, S. Klein, R. Carius, H. Stiebig, X. Geng, F. Finger, Open circuit voltage improvement of high-deposition-rate microcrystalline silicon solar cells by hot wire interface layers, Appl. Phys. Lett. 87 (7) (2005) 073503.

[5] D. E. Carlson, C. R. Wronski, Amorphous Silicon Solar Cell, Appl. Phys. Lett. 28 (11) (1976) 671-673.

[6] V. Smirnov, A. Lambertz, B. Grootoonk, R. Carius, F. Finger, Microcrystalline silicon oxide $\left(\mu \mathrm{c}-\mathrm{SiO}_{x}: \mathrm{H}\right)$ alloys: A versatile material for application in thin film silicon single and tandem junction solar cells, Journal of Non-crystalline Solids 358 (17) (2012) 1954-1957, doi: 10.1016/j.jnoncrysol.2011.12.019.

[7] F. Meillaud, A. Billet, C. Battaglia, M. Boccard, G. Bugnon, P. Cuony, M. Charrière, M. Despeisse, L. Ding, JordiEscarre-Palou, S. Hänni, L. Löfgren, S. Nicolay, G. Parascandolo, M. E. Stckelberger, C. Ballif, Latest Developments of High-Efficiency Micromorph Tandem Silicon 
Solar Cells Implementing Innovative Substrate Materials and Improved Cell Design, IEEE Journal of Photovoltaics 2 (2012) 236-240.

[8] M. A. Green, K. Emery, Y. Hishikawa, W. Warta, Solar cell efficiency tables (version 37), Prog. Photovoltaics 19 (1) (2011) 84-92.

[9] F. Finger, L. B. Neto, R. Carius, T. Dylla, S. Klein, Paramagnetic defects in undoped microcrystalline silicon, phys. stat. sol. (c) 1 (5) (2004) 1248-1254.

[10] T. Umeda, S. Yamasaki, J. Isoya, K. Tanaka, Electron-spin-resonance center of dangling bonds in undoped a-Si:H, Phys. Rev. B 59 (7) (1999) 4849-4857.

[11] J. Müller, F. Finger, R. Carius, H. Wagner, Electron spin resonance investigation of electronic states in hydrogenated microcrystalline silicon, Phys. Rev. B 60 (16) (1999) 11666-11677.

[12] W. Fuhs, Recombination and transport through localized states in hydrogenated amorphous and microcrystalline silicon, J. Non-Cryst. Solids 354 (19-25) (2008) 2067-2078.

[13] M. Fehr, A. Schnegg, B. Rech, K. Lips, O. Astakhov, F. Finger, G. Pfanner, C. Freysoldt, J. Neugebauer, R. Bittl, C. Teutloff, Combined multifrequency EPR and DFT study of dangling bonds in a-Si:H, Phys. Rev. B 84 (2011) 245203.

[14] J. Schmidt, I. Solomon, Modulation De La Photoconductivite Dans Le Silicium a Basse Temperature Par Resonance Magnetique Electronique 
Des Impuretes Peu Profondes, Compt. Rend. Acad. Sci. B 263 (2) (1966) $169-172$.

[15] M. Stutzmann, M. S. Brandt, M. W. Bayerl, Spin-dependent processes in amorphous and microcrystalline silicon: a survey, J. Non-Cryst. Solids 266-269 (2000) 1-22.

[16] C. Boehme, K. Lips, Time domain measurement of spin-dependent recombination, Appl. Phys. Lett. 79 (26) (2001) 4363-4365.

[17] C. Boehme, K. Lips, Theory of time-domain measurement of spin-dependent recombination with pulsed electrically detected magnetic resonance, Physical Review B 68 (24) (2003) 245105, doi: 10.1103/PhysRevB.68.245105.

[18] J. Behrends, A. Schnegg, C. Boehme, S. Haas, H. Stiebig, F. Finger, B. Rech, K. Lips, Recombination and transport in microcrystalline pin solar cells studied with pulsed electrically detected magnetic resonance, Journal of Non-crystalline Solids 354 (19-25) (2008) 2411-2415, doi: 10.1016/j.jnoncrysol.2007.09.086.

[19] J. Behrends, A. Schnegg, M. Fehr, A. Lambertz, S. Haas, F. Finger, B. Rech, K. Lips, Electrical detection of electron spin resonance in microcrystalline silicon pin solar cells, Philosophical Magazine 89 (28-30) (2009) 2655-2676, doi:10.1080/14786430903008472.

[20] H. Huebl, F. Hoehne, B. Grolik, A. R. Stegner, M. Stutzmann, M. S. Brandt, Spin echoes in the charge transport through phosphorus donors in silicon, Phys. Rev. Lett. 100 (17) (2008) 177602. 
[21] F. Hoehne, H. Huebl, B. Galler, M. Stutzmann, M. S. Brandt, SpinDependent Recombination between Phosphorus Donors in Silicon and $\mathrm{Si} / \mathrm{SiO} 2$ Interface States Investigated with Pulsed Electrically Detected Electron Double Resonance, Phys. Rev. Lett. 104 (2010) 046402.

[22] F. Hoehne, L. Dreher, H. Huebl, M. Stutzmann, M. S. Brandt, Electrical Detection of Coherent Nuclear Spin Oscillations in Phosphorus-Doped Silicon using Pulsed ENDOR, Phys. Rev. Lett. 106 (2011) 187601.

[23] M. Fehr, J. Behrends, S. Haas, B. Rech, K. Lips, A. Schnegg, Electrical detection of electron-spin-echo envelope modulations in thinfilm silicon solar cells, Physical Review B 84 (19) (2011) 193202, doi: 10.1103/PhysRevB.84.193202.

[24] D. J. Lepine, Spin-Dependent Recombination on Silicon Surface, Phys. Rev. B 6 (2) (1972) 436-441.

[25] G. W. Morley, D. R. McCamey, H. A. Seipel, L. C. Brunel, J. van Tol, C. Boehme, Long-Lived Spin Coherence in Silicon with an Electrical Spin Trap Readout, Phys. Rev. Lett. 101 (20) (2008) 207602.

[26] C. C. Lo, J. Bokor, T. Schenkel, A. M. Tyryshkin, S. A. Lyon, Spindependent scattering off neutral antimony donors in ${ }^{28}$ Si field-effect transistors, Appl. Phys. Lett. 91 (24) (2007) 242106.

[27] C. C. Lo, V. Lang, R. E. George, J. J. L. Morton, A. M. Tyryshkin, S. A. Lyon, J. Bokor, T. Schenkel, Electrically Detected Magnetic Resonance of Neutral Donors Interacting with a Two-Dimensional 
Electron Gas, Physical Review Letters 106 (20) (2011) 207601, doi: 10.1103/PhysRevLett.106.207601.

[28] M. Bennati, T. F. Prisner, New developments in high field electron paramagnetic resonance with applications in structural biology, Reports On Progress In Physics 68 (2) (2005) 411-448, doi:10.1088/00344885/68/2/R05.

[29] D. Kaplan, I. Solomon, N. F. Mott, Explanation of Large SpinDependent Recombination Effect in Semiconductors, Journ. de Phys. Lettr. 39 (4) (1978) L51-L54.

[30] B. Rech, T. Roschek, T. Repmann, J. Müller, R. Schmitz, W. Appenzeller, Microcrystalline silicon for large area thin film solar cells, Thin Solid Films 427 (1-2) (2003) European Mat Res Soc, doi:10.1016/S00406090(02)01210-5.

[31] M. Luysberg, P. Hapke, R. Carius, F. Finger, Structure and growth of hydrogenated microcrystalline silicon: Investigation by transmission electron microscopy and Raman spectroscopy of films grown at different plasma excitation frequencies, Philosophical Magazine A-physics of Condensed Matter Structure Defects and Mechanical Properties 75 (1) (1997) 31-47.

[32] S. Haas, A. Gordijn, H. Stiebig, High speed laser processing for monolithical series connection of silicon thin-film modules, Progress In Photovoltaics 16 (3) (2008) 195-203, doi:10.1002/pip.792. 
[33] A. Stesmans, G. Vangorp, Novel Method For Accurate g-Measurements In Electron-spin Resonance, Review of Scientific Instruments 60 (9) (1989) 2949-2952, doi:10.1063/1.1140633.

[34] V. Lang, C. C. Lo, R. E. George, S. A. Lyon, J. Bokor, T. Schenkel, A. Ardavan, J. J. L. Morton, Electrically detected magnetic resonance in a W-band microwave cavity, Review of Scientific Instruments 82 (3) (2011) 034704, doi:10.1063/1.3557395.

[35] E. L. Dane, T. Maly, G. T. Debelouchina, R. G. Griffin, T. M. Swager, Synthesis of a BDPA-TEMPO Biradical, Organic Letters 11 (9) (2009) 1871-1874, doi:10.1021/ol9001575.

[36] C. Boehme, K. Lips, The ultra-sensitive electrical detection of spinRabi oscillation at paramagnetic defects, Physica B-condensed Matter 376 (2006) 930-935, doi:10.1016/j.physb.2005.12.231.

[37] J. R. Knutson, D. G. Walbridge, L. Brand, Decay-associated Fluorescence-spectra and the Heterogeneous Emission of Alcoholdehydrogenase, Biochemistry 21 (19) (1982) 4671-4679, doi: 10.1021/bi00262a024.

[38] S. Hasegawa, S. Narikawa, Y. Kurata, Electron-spin-resonance and Electrical-properties of P-doped Microcrystalline Si, Philosophical Magazine B-physics of Condensed Matter Statistical Mechanics Electronic Optical and Magnetic Properties 48 (5) (1983) 431-447, doi: $10.1080 / 13642818308228569$. 
[39] F. Finger, C. Malten, P. Hapke, R. Carius, R. Flückiger, H. Wagner, Free-electrons and Defects In Microcrystalline Silicon Studied By Electron-spin-resonance, Philosophical Magazine Letters 70 (4) (1994) 247-254, doi:10.1080/09500839408240982.

[40] W. Fuhs, P. Kanschat, K. Lips, Bandtails and defects in microcrystalline silicon ( $\mu$ c-Si:H), Journal of Vacuum Science \& Technology B 18 (3) (2000) 1792-1795, doi:10.1116/1.591473.

[41] M. Kondo, T. Nishimiya, K. Saito, A. Matsuda, Light induced phenomena in microcrystalline silicon, Journal of Non-crystalline Solids 227 (1998) 1031-1035, doi:10.1016/S0022-3093(98)00276-2.

[42] F. Finger, J. Müller, C. Malten, R. Carius, H. Wagner, Electronic properties of microcrystalline silicon investigated by electron spin resonance and transport measurements, Journal of Non-crystalline Solids 266 (2000) 511-518, doi:10.1016/S0022-3093(99)00802-9.

[43] M. M. de Lima, P. C. Taylor, S. Morrison, A. LeGeune, F. C. Marques, ESR observations of paramagnetic centers in intrinsic hydrogenated microcrystalline silicon, Physical Review B 65 (23) (2002) 235324, doi: 10.1103/PhysRevB.65.235324.

[44] B. M. George, J. Behrends, A. Schnegg, T. F. Schulze, M. Fehr, L. Korte, B. Rech, K. Lips, M. Rohrmüller, E. Rauls, W. G. Schmidt, U. Gerstmann, Atomic Structure of Interface States in Silicon Heterojunction Solar Cells, Phys. Rev. Lett. 
110 (2013) 136803, doi:10.1103/PhysRevLett.110.136803, URL http://link.aps.org/doi/10.1103/PhysRevLett.110.136803.

[45] J. H. Zhou, S. D. Baranovskii, S. Yamasaki, K. Ikuta, M. Kondo, A. Matsuda, K. Tanaka, Transport properties of microcrystalline silicon at low temperatures, Semiconductors 32 (8) (1998) 807-811, doi: 10.1134/1.1187511.

[46] L. Houben, M. Luysberg, P. Hapke, R. Carius, F. Finger, H. Wagner, Structural properties of microcrystalline silicon in the transition from highly crystalline to amorphous growth, Philosophical Magazine A-physics of Condensed Matter Structure Defects and Mechanical Properties 77 (6) (1998) 1447-1460.

[47] Q. Y. Ye, R. Tsu, E. H. Nicollian, Resonant Tunneling Via Microcrystalline-silicon Quantum Confinement, Physical Review B 44 (4) (1991) 1806-1811, doi:10.1103/PhysRevB.44.1806.

[48] N. Nestle, G. Denninger, M. Vidal, C. Weinzierl, K. Brunner, K. Eberl, K. von Klitzing, Electron spin resonance on a two-dimensional electron gas, Physical Review B 56 (8) (1997) R4359-R4362.

[49] R. N. Pereira, A. R. Stegner, T. Andlauer, K. Klein, H. Wiggers, M. S. Brandt, M. Stutzmann, Dielectric screening versus quantum confinement of phosphorus donors in silicon nanocrystals investigated by magnetic resonance, Physical Review B 79 (16) (2009) 161304, doi: 10.1103/PhysRevB.79.161304. 
[50] C. Freysoldt, G. Pfanner, J. Neugebauer, The dangling-bond defect in amorphous silicon: Statistical random versus kinetically driven defect geometries, Journal of Non-crystalline Solids 358 (17) (2012) 20632066, doi:10.1016/j.jnoncrysol.2011.12.090.

[51] U. Gerstmann, M. Rohrmüller, F. Mauri, W. Schmidt, Ab initio g-tensor calculation for paramagnetic surface states: hydrogen adsorption at $\mathrm{Si}$ surfaces, Phys. Status Solidi C 7 (19) (2010) 157160.

[52] W. R. Hagen, D. O. Hearshen, R. H. Sands, W. R. Dunham, A statistical theory for powder electron paramagnetic resonance In distributed systems, Journal of Magnetic Resonance 61 (2) (1985) 220-232, doi: 10.1016/0022-2364(85)90077-0.

[53] J. R. Pilbrow, Lineshapes in frequency-swept and field-swept EPR for Spin 1/2, Journal of Magnetic Resonance 58 (2) (1984) 186-203, doi: 10.1016/0022-2364(84)90209-9.

[54] D. R. McCamey, G. W. Morley, H. A. Seipel, L. C. Brunel, J. van Tol, C. Boehme, Spin-dependent processes at the crystalline $\mathrm{Si}-\mathrm{SiO}(2)$ interface at high magnetic fields, Physical Review B 78 (4) (2008) 045303, doi:10.1103/PhysRevB.78.045303. 